\title{
Efficacy Analysis of Gastric Coronary Venous TH Glue Embolization with Splenectomy for Treating Cirrhotic Portal Hypertension
}

\author{
Zhenyu Li ${ }^{1, \#}$, Xi Wang ${ }^{2, \#}$, Jinwei Chen ${ }^{1}$, Zusheng Zang ${ }^{1}$, Feng Zhou ${ }^{1}$, Liqin Shi $^{1}$, \\ $\mathrm{Li} \mathrm{Li}^{3}$, Chengwei Chen ${ }^{1}$, Xiaojin Wang ${ }^{*}$, Yinpeng Jin ${ }^{3 *}$ and Qingchun $\mathrm{Fu}^{3 *}$ \\ ${ }^{1}$ Shanghai Liver Diseases Research Center, The 85th Hospital of PLA, Shanghai 200235, China; ${ }^{2}$ Department of Infection, Affiliated \\ Hospital of Guizhou Medical University, 550004, Guiyang, Guizhou Province, China; ${ }^{3}$ Shanghai Public Health Clinical Center, Fudan

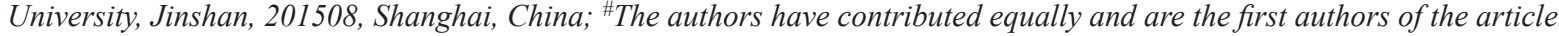

\begin{abstract}
Background and aims: To evaluate the effects of gastric coronary venous embolization with TH glue (developercontaining octyl- $\alpha$-cyanoacrylate) in combination with splenectomy for the treatment of cirrhotic portal hypertension and gastroesophageal varices.
\end{abstract}

Methods: From April 2002 to July 2016, 81 patients with cirrhotic portal hypertension who underwent this procedure were subject to perioperative (within 2 weeks), short-term (within 2 weeks to 1 month) and long-term (1 month thereafter) efficacy analyses. Complications, rebleeding rate, and long-term survival rate were evaluated.

Results: No patients developed embolism caused by TH glue ectopia. Eleven patients experienced perioperative complications, including high esophageal expenditure blood (1\%), subphrenic effusion (1\%) and abdominal infection (1\%), which affected one case each respectively. Pulmonary infection (2\%) and portal system thrombosis (2\%) affected two cases respectively. There were 4 patients who experienced ascites (5\%). All patients had small amounts of melena and were healed after conservative medical treatment. The 1-, 3-, 5- and 10-year postoperative rebleeding rates were $4.9 \%, 8.6 \%, 11.1 \%$ and $18.5 \%$ respectively. The 1-, 3-, 5- and 10 -year postoperative survival rates were $97.5 \%, 92.6 \%$, $90.1 \%$ and $80.2 \%$ respectively. No hepatic encephalopathy occurred within 1 year after operation in any case.

Conclusions: The postoperative rebleeding rate was lower than that reported in the literature and the subjects achieved good perioperative, short-term and long-term effects. The method of operation in the treatment of cirrhotic portal hypertension and gastroesophageal varices is characterized by a good safety profile, less invasiveness, rapid postoperative recovery, and a lower rebleeding rate than other devascularization procedures. Thus, it is an option that can be first considered by patients requiring emergency surgery to stop bleeding or patients with poor liver function, and even some patients with Child-Pugh grade C.

Keywords: Portal hypertension; Embolization; TH glue.

Received: April 24, 2019; Revised: May 16, 2019; Accepted: May 17, 2019

*Correspondence to: Qingchun Fu, Shanghai Public Health Clinical Center, Fudan University, Jinshan, 201508, Shanghai, China. E-mail: qcfu85@163.com. Yinpeng Jin, Shanghai Public Health Clinical Center, Fudan University, Jinshan, 201508, Shanghai, China. E-mail: 1182008211@qq.com. Xiaojin Wang, Shanghai Liver Diseases Research Center, The 85th Hospital of PLA, Shanghai 200235, China. E-mail: drwxj85@126.com.

How to cite this article: Li Z, Wang X, Chen J, Zang Z, Zhou F, Shi L, Li L, Chen C, Wang X, Jin Y, Fu Q. Efficacy Analysis of Gastric Coronary Venous TH Glue Embolization with Splenectomy for Treating Cirrhotic Portal Hypertension. Exploratory Research and Hypothesis in Medicine 2019;4(4):69-75. doi: 10.14218/ ERHM.2019.00010.
Introduction

Portal hypertension is a clinical syndrome affecting patients with or without cirrhosis, in which hemodynamic changes in the portosystemic circulation lead to complications such as variceal bleeding, ascites, and portosystemic encephalopathy. ${ }^{1-3}$ Upper gastrointestinal bleeding caused by esophageal varices is its most serious complication, with an annual bleeding rate of $5-15 \%$ and a rebleeding rate of $60 \%$ within 1 year. ${ }^{4}$ The surgical treatment of portal hypertension primarily aims to prevent esophageal variceal bleeding. The surgical mode is generally divided into shunt and devascularization, with their own unique theoretical bases and different efficacies. Shunting can significantly reduce portal pressure, with good hemostatic effects and a low postoperative 
bleeding rate, but it has great impact on liver blood flow and a high incidence rate of hepatic encephalopathy. Devascularization has a good proximate hemostatic effect but a high postoperative rebleeding rate and incomplete blockade of blood flow (the high and ectopic high esophageal branches are often difficult to handle properly); the non-satisfactory treatment of gastric wall muscular layers and submucosal varicose veins, and the problems in processing postoperative portal vein thrombosis have restricted the development of devascularization. ${ }^{5}$

The present procedure of gastric coronary venous embolization with $\mathrm{TH}$ glue in combination with splenectomy for the treatment of cirrhotic portal hypertension and gastroesophageal varices is currently in routine use for devascularization. It is characterized by a good safety profile, less invasiveness, and rapid postoperative recovery. However, the rebleeding rate of the operation has been high, and the short-term and long-term efficacies have been less studied.

From 2002 to 2016, we performed splenectomy and portoazygous devascularization on 109 patients with cirrhotic portal hypertension and gastroesophageal varices. In this study, we used different methods of disconnection surgery, focusing on the treatment of gastric coronary venous TH embolization combined with splenectomy, making some improvements to this method, and we evaluated the short-term and long-term efficacy. The devascularization mode was gastric coronary venous ligation (non-TH glue; adopted by 12 patients), pericardial devascularization (adopted by 10 patients), and TH gastrointestinal coronary venous embolization (adopted by 87 patients) (April 2002 to July 2015); of the 87 patients receiving TH glue embolization, 81 were followed up (with a follow-up rate of $93.1 \%$ ).

The 81 patients with cirrhotic portal hypertension and gastroesophageal varices treated by this procedure were subject to shortterm and long-term efficacy analyses; with perioperative complications, rebleeding rate and survival rate etc. as the indicators, an evaluation was performed in terms of the efficacy and safety of this surgical mode in treating cirrhotic portal hypertension with gastroesophageal varices.

\section{Methods}

\section{Participants}

From April 2002 to July 2016, 81 patients with cirrhosis and portal hypertension were treated with TH glue embolization and the curative effect was evaluated. All patients provided written informed consent. In this study, no measurement of portal vein pressure was carried out. The clinical manifestations of patients with liver cirrhosis complicated with portal hypertension, such as splenomegaly, ascites and esophageal varices, were comprehensively analyzed. The mean follow-up duration of the 81 patients was 9.3 \pm 2.8 years. There were 57 men and 24 women (ratio of $2.38: 1$ ), with a mean age of $48.7 \pm 10.6$ years. Among them, 22 patients had a history of upper gastrointestinal bleeding before operation, with moderate and severe esophageal varices confirmed by gastroscopy. Eight patients underwent emergency surgery.

Etiological diagnoses included 72 cases of hepatitis B-related decompensated cirrhosis (the chronic hepatitis B patients received antiviral treatment according to China's prevention and treatment guidelines), 1 case of hepatitis C-related decompensated cirrhosis, 3 cases of alcoholic decompensated cirrhosis, 1 case of schistosomiasis cirrhosis, 1 case of autoimmune hepatitis complicated by primary decompensated biliary cirrhosis, 1 case of primary decompensated biliary cirrhosis, and 2 cases of cirrhosis of an unknown cause.

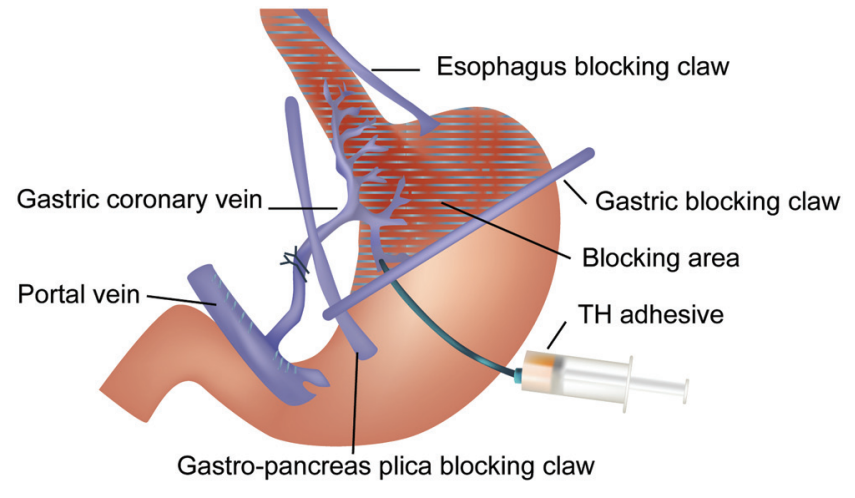

Fig. 1. Schematic diagram of gastric venous embolization with $\mathrm{TH}$ glue.

Liver function assessment (Child-Pugh score) classified 42 cases as grade A, 35 cases as Child grade B, and 4 cases as Child grade C. In 8 cases of emergency operation, in terms of the liver functional classification, 5 cases were grade $\mathrm{B}$ and 3 cases were grade $\mathrm{C}$.

This study design is mainly retrospective and did not involve ethical issues. This study conformed to the ethical norms of the Helsinki Declaration.

\section{Surgical methods}

All patients were treated with endotracheal intubation and balanced anesthesia; an "L"-shaped incision was made posterior to the left costal margin to open the gastrocolic ligament. First, the splenic artery was dissociated and ligated, and the splenic vein was protected. At the same time, the spleen was squeezed to help the spleen's blood flow out through the splenic vein. When separating the splenogastric ligament, attempts were made to perform the ligature at a site near the spleen. The spleen was removed by in situ method, ${ }^{6}$ and the spleen pedicle and the pancreatic tail stump were subject to intermittent interlocking, suturing and ligation by two layers with No. 7 line. Peri-gastric vessels were dissociated and disjuncted, and after simultaneously separating and ligating the gastric coronal artery and the left gastric artery, the embolic area was embolized using the method of clamping the "enclosed" embolic area and applying 8-10 mL of TH glue (octyl$\alpha$-cyanoacrylate embolic glue; Guangzhou Baiyun Medical Glue Co., Ltd., China) (Fig. 1).

\section{Statistical analysis}

All analyses were performed using Excel 2003 (Microsoft, WA, USA). The variables were expressed as mean \pm standard deviation. The comparison of two independent groups was performed using Student's $t$-test. A $p$-value of 0.05 or less was considered to be statistically significant.

\section{Results}

\section{Main endpoints of efficacy observation}

Rebleeding

One patient (1\%) had high esophageal expenditure during the 


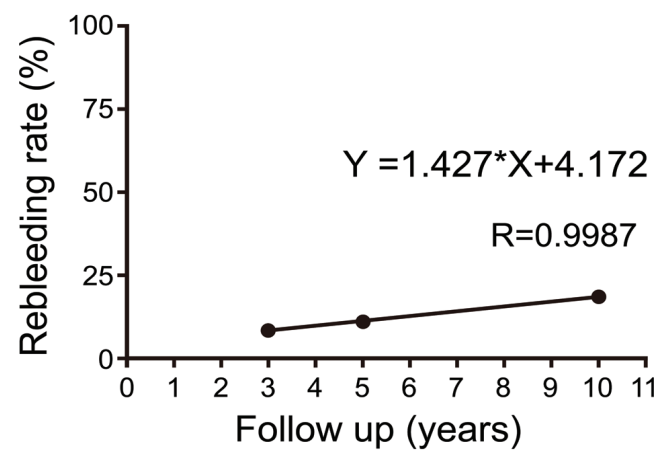

Fig. 2. Follow-up of postoperative rebleeding rate.

perioperative period. There were 2 cases of postoperative gastrointestinal bleeding from 1 month to 1 year after surgery. A small amount of black feces appeared in both patients and was cured by conservative treatment. In 7 cases, rebleeding was reported within the 3-year follow-up period, 9 cases reported within 5 years and 15 cases within 10 years, and the rebleeding rate was $8.6 \%, 11.1 \%$ and $18.5 \%$ within 3,5 and 10 years respectively. Six of these patients died of gastrointestinal bleeding, and the conditions of the remaining patients were controlled by conservative medical treatment. Further analysis of the relationship between rebleeding rate and follow-up years showed a significantly positive correlation between them. The correlation coefficient $\mathrm{R}$ was $0.9987(p<0.05)$ (Fig. 2).

\section{Mortality/survival rate}

There were 19 cases of death (including 1 case of death caused by septic shock during the perioperative period), 7 cases of gastrointestinal bleeding, 6 cases of liver cancer, 1 case of hepatic encephalopathy, 3 cases of hepatorenal syndrome, and 1 case of renal tubular acidosis. The 1-, 3-, 5- and 10-year postoperative survival rates were $97.5 \%, 92.6 \%, 90.1 \%$ and $80.2 \%$ respectively. Further analysis of the relationship between survival rate and follow-up years showed a significantly positive correlation between them. The correlation coefficient $\mathrm{R}$ was $0.9987(p<0.05)$ (Fig. 3).

\section{Secondary endpoint of efficacy observation}

Emergency operation

Of the 8 patients received an emergency surgery, 5 were of ChildPugh grade B and 3 of grade C. For all, the bleeding was stopped immediately, with a hemostasis success rate of $100 \%$. Two of the patients died of liver cancer within 2 years after the surgery, and the subsequent follow-up showed that the remaining 6 patients survived well.

\section{Patients of Child-Pugh grade C}

Four patients in this group (both diagnosed with hepatitis B cirrhosis) went through the perioperative period smoothly and no further bleeding was observed, even after long-term follow-up. Two of the patients died of liver cancer (within 2 years), and the subsequent follow-up showed that the other 2 patients survived well (the follow-up duration was 9 and 11 years respectively).

\section{Spleen heat and corresponding treatment}

Before 2012, 9 (12.3\%) patients experienced spleen heat of varying degrees (continuous fever more than 10 days, with a body temperature of above $38^{\circ} \mathrm{C}$ ) within 2 weeks to 1 month, and the body temperature decreased to normal after 4-week symptomatic treatment. From day 3 after the initiation of operation in 2012, low-molecular weight heparin calcium was conventionally administered as the anticoagulant therapy, involving a total of 8 cases, and no spleen heat occurred again.

\section{Treatment of portal vein thrombosis}

In the perioperative period, 2 cases of portal vein thrombosis were detected by early monitoring of B-ultrasound, and the thrombi disappeared within 3 months to 6 months after anticoagulant therapy (i.e. heparin). Among the 73 patients before 2012, cavernous transformation of the portal vein was reported in 16 after long-term postoperative follow-up, and it was speculated that

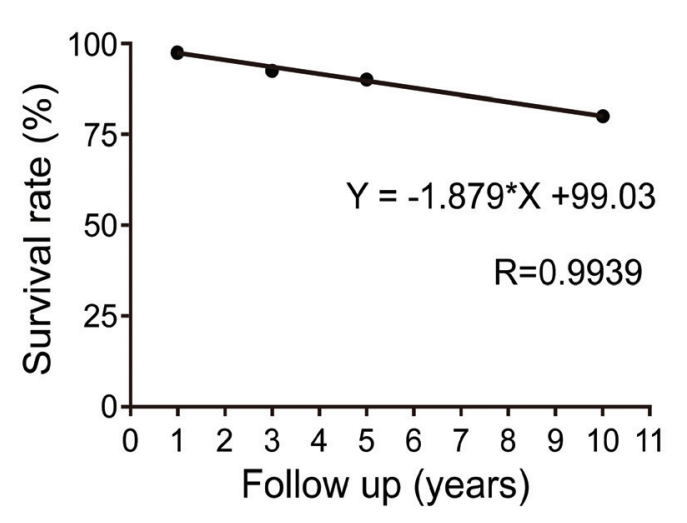

\section{Cause of death}

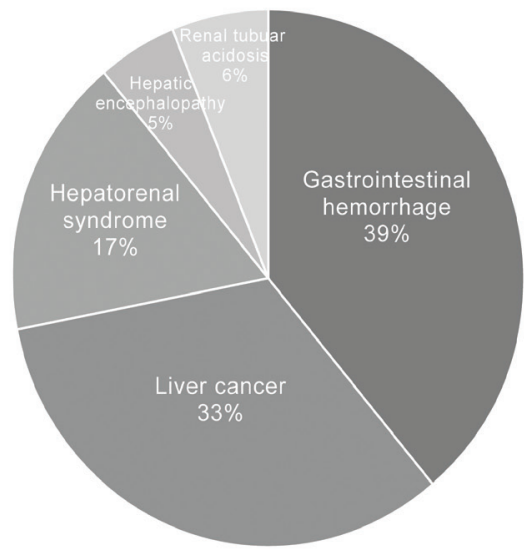

Fig. 3. Follow-up of postoperative survival rate and analysis of cause of death. 
Table 1. Outcome of postoperative liver function and survival rate

\begin{tabular}{llllll}
\hline \multirow{2}{*}{ Grade } & $\boldsymbol{n}$ & \multicolumn{3}{c}{ Outcome of liver function } \\
\cline { 3 - 6 } & & Grade A & Grade B & Grade C & 5-year survival rate \\
\hline Child-Pugh A & 42 & $27(64.3 \%)$ & $9(21.4 \%)$ & $6(14.3 \%)$ & $95.2 \%$ \\
Child-Pugh B & 35 & $23(65.7 \%)$ & $8(22.9 \%)$ & $4(11.4 \%)$ & $88.6 \%$ \\
Child-Pugh C & 4 & $2(50.0 \%)$ & $0(0.0 \%)$ & $2(50.0 \%)$ & $50.0 \%$ \\
\hline
\end{tabular}

there might be portal vein thrombosis during the perioperative period. After conventional anticoagulation and early B-ultrasound monitoring in 2012, only two cases of early portal thrombosis were found; after intensified anticoagulant therapy, thrombi disappeared within 3 months to 6 months, and long-term follow-up showed that none of the patients developed cavernous transformation of the portal vein.

\section{Postoperative liver function evaluation}

All patients were followed up for 5 years to re-evaluate their liver function (Child-Pugh score). Of the 42 patients whose liver function was grade A before operation, 27 had grade A, 9 had grade B and 6 had grade $\mathrm{C}$, and $2(4.8 \%)$ died within 5 years. Among the 35 patients whose liver function was grade $\mathrm{B}$ before operation, 2 improved to grade $\mathrm{A}$ after 5 years and 2 remained as grade $\mathrm{C}$, and 2 patients $(50 \%)$ died within 5 years (hepatocellular carcinoma). According to the above data, 60 out of 81 patients (74\%) showed that their liver function was maintained or improved after operation (Table 1).

\section{Hepatic encephalopathy}

No hepatic encephalopathy occurred within 1 year after operation. Two cases $(2.5 \%)$ of hepatic encephalopathy were reported within 5 years after operation, and two other cases $(2.5 \%)$ of hepatic encephalopathy were reported within 5-10 years after. Except for the 1 case that died of hepatic encephalopathy 4 years after the operation, the conditions of the remaining cases were controlled by low-protein diet, showed resistance to coma, and received laxative treatment.

\section{Other symptoms}

In the perioperative period, there was 1 case of subphrenic effusion, 2 cases of pulmonary infection, 2 cases of portal system thrombosis, 4 cases of ascites, and 1 case of intraperitoneal infection, who died of septic shock 2 weeks after operation. The case of ascites subsided after intensified diuresis and protein-supplementary therapies within 1 month. Four cases developed ascites, and the ascites subsided within 1 month after the administration of intensified diuresis, within a 2 -week to 1 -month period.

\section{Discussion}

At present, the surgical methods of cirrhotic portal hypertension are mainly categorized into shunting and devascularization. However, since the decrease of hepatic perfusion after shunting can further impair liver function and even lead to high mortality due to he- patic encephalopathy, it is seldom chosen by surgeons. ${ }^{7-9}$ While it is stated that the recently developed "selective shunt theoretically selectively reduces the pressure of gastroesophageal varices without affecting the portal-vein-to-liver perfusion", in fact, about $60 \%$ of patients have little or no hepatopetal flow after shunting. ${ }^{10}$ Yin et al. ${ }^{11}$ conducted a meta-analysis of the therapeutic effect of portal hypertension after operation, and found that there was no significant difference in the recurrence rate of bleeding and complications of hepatic encephalopathy between selective and non-selective shunts. In China, most of the cirrhosis cases are caused by posthepatitis status, so the indications for shunting are very limited, and the main method of cirrhotic portal hypertension is devascularization.

After decades of clinical practice and research, the role and status of devascularization in the treatment of gastric varices bleeding with portal hypertension has been fully established. ${ }^{12-14}$ Gastric fundus venous hemorrhage can occur in a relatively wide area from the fundus of the stomach to the lower esophagus. Whether the upper digestive tract bleeds after devascularization depends mainly on the blockage of blood flow. In order to effectively control variceal bleeding and maintain effective liver blood supply. We currently believe that gastric coronary venous TH embolization combined with splenectomy is the appropriate option.

The occurrence of upper gastrointestinal tract rebleeding indicates a poor prognosis. In patients with cirrhotic portal hypertension, the communicating branch of the portal vein is patent and the traffic branch is not only present in the outer layer of the esophagogastric wall but also in the esophagogastric muscular layer and submucosa, mainly derived from the abnormal blood flow of the gastric coronary vein and flowing into the lower varicose vein of the esophagus through the esophageal branch and into the gastric submucosal vein through the gastric branch. At the same time, the submucosal vein of the gastric fundus also receives abnormal blood flow from the short gastric vein and the venous posterior vein. Satisfying devascularization effects can be achieved after blocking all the abnormal flow routes interior and exterior to the esophageal wall.

The advantage of gastric coronary venous $\mathrm{TH}$ embolization combined with splenectomy in controlling bleeding is mainly due to a wide range of embolization, which reaches 10-12 cm superior to the cardia and fully meets the scope of blocking required by sugiura. ${ }^{15}$ The injection of TH gel embolic agent through the coronary vein can simultaneously block the blood flow of the entire esophageal wall, and the devascularization effect is relatively thorough. During the gastric coronary venous TH glue embolization, it is unnecessary to separate all the blood vessels around the cardia but it can block all the blood vessels around the cardia, including the high esophageal branch and the ectopic high esophageal branch, so the range of portoazygous devascularization is relatively thorough. ${ }^{16}$ The devascularization effect is safer, avoiding extensive separation of the pericardia areas and causing small surgical intervention to the surrounding areas. TH gel embolic agent, as a permanent embolic agent, is completely solidified within 10-15 s after being injected into the blood vessel. It can ensure the persis- 
tence of the devascularization and embolize the abnormal blood flow interior and exterior to the esophagogastric wall as well, exerting effects similar to those of blocking the pericardia blood vessels and transversely discontinuing the esophageal blood vessels.

Liver cirrhosis is usually accompanied by hypersplenism. Splenectomy corrects the state of the flow and has a positive effect on reducing postoperative rebleeding. Hermann et al. ${ }^{17}$ retrospectively analyzed the clinical materials collected by their institution for 50 years. It was found that the long-term (5-10 years) rebleeding rate after devascularization was as high as $40-50 \%$. In this group, the rebleeding rates after the $\mathrm{TH}$ gel embolization were $8.6 \%, 11.1 \%$ and $18.5 \%$ at 3,5 and 10 years after the operation, respectively. Compared with other devascularization procedures, it showed some advantages, especially in the mid-term and longterm bleeding rates. The injection of TH gel embolic agent through the coronary vein can simultaneously block the blood flow of the entire esophageal wall, and the devascularization effect is relatively thorough.

To further reduce the bleeding rate during or after operation, we have also made some modifications in the surgical mode: anatomy in situ. (1) The previous recommendation for the surgery is that the spleen be lifted out or partially removed from the abdominal cavity before the treatment of spleen pedicle. Our experience is to perform the anatomy in situ as much as possible, not lifting the spleen out prematurely. According to the principle of "first superficial and then deep, first easy and then difficult", the spleen is naturally easy to remove after the complete separation of the perisplenic ligaments, blood vessels, and other adhesive tissues. The advantage lies in that this approach can avoid tearing of the fragile perisplenic blood vessels that could be caused by the traction of the perisplenic vessels and the changes in the normal anatomic structure. (2) The splenic artery is first ligated during the operation. Asoglu et al. ${ }^{18}$ advocated the ligation of the splenic artery during the early stage of the surgery, because this was safe, effective and feasible. It was shown to reduce intraoperative bleeding, facilitate anatomy of the spleen, and to not increase the operation duration. During the operation, the splenic artery was first ligated. Due to the splenic venous return, the spleen would become smaller and softer, which was equivalent to autologous blood transfusion. (3) After the spleen resection, the splenic pedicle and the pancreatic tail were subject to "double-layer interlocking, suturing and ligation", which can effectively reduce the occurrence of relevant complications, such as bleeding, pancreatic leak and subphrenic infection caused by clot detachment after the ligation of large tissue clots in this area. However, the operation cannot fully alleviate portal hypertension. The rates of rebleeding in the 5 and 10 years after the operation were $11.1 \%$ and $18.5 \%$ respectively. In terms of how to solve this problem, we need to carry out further research on the surgical methods, skills, and innovation.

A wide range of TH gel embolization has less physiological intervention. In this way, the procedure saves the trouble of discontinuing the pericardia blood vessels and the necessity of disjuncting the gastric vagus nerve, maintaining good esophageal pressure and gastric motility, and preventing gastric retention due to gastric vagus nerve injury; preventing the obstruction of collateral circulation increased the blood flow of hepatic artery was beneficial to the recovery of liver function. The operation is simple, the damage to the patient is small, and the surgical indications are wide. ${ }^{18}$ In the 4 patients with Child grade $\mathrm{C}$ who underwent gastric coronary venous TH embolization combined with splenectomy, their conditions were stable in the perioperative period, without any complications related to surgery and at postoperative follow-up (more than 2 years) showed no gastrointestinal bleeding occurrences and good therapeutic efficacy was obtained. For compensatory cir- rhosis patients who need emergency surgery to stop bleeding and those with a poor liver function (of Child grade C), gastric coronary venous TH embolization and splenectomy can be considered as a priority due to its low invasiveness and good efficacy.

After splenectomy in combination with TH gel embolization, portal thrombosis may arise - causing complications such as aggravating portal hypertension and loss of the chance of liver transplant surgery. In order to reduce the incidence of portal vein embolism, the operation was improved by our group. During the operation, the three intestinal clips were used to clamp to the gastropancreatic fold (inferiorly reaching the posterior aspect of the cardia), the fundus, and the vertical part of hepatogastric ligament, which can effectively prevent the occurrence of misembolism of TH gel ectopia (liver, lung, etc.). We did not find any ectopic embolism intraoperatively or postoperatively. Since 2012, we have been administering the patients $0.4 \mathrm{~mL}$ of low-molecular weight heparin on days 3 to 5 day after surgery, given as subcutaneous injection, q12h, for 2 weeks. Every 2 weeks thereafter, the lowmolecular weight heparin was withdrawn and replaced by the oral warfarin for anticoagulant monotherapy. Low-molecular weight heparin does not prolong bleeding time, its preventive dose does not significantly change prothrombin time and activated partial thromboplastin time, and it can exert persistent anticoagulant effects through subcutaneous injection and can be used continuously for a long time; warfarin can inhibit the formation of prothrombin and other coagulation factors. The blood flow of the portal vein system and routine blood test indicators were examined by color Doppler ultrasound at 1 week, 2 weeks and 1 month after the operation. According to the international normalized ratio, the warfarin dosage was adjusted to $2-5 \mathrm{mg}$ qd. Thrombi disappeared within 3-6 months, and only 2 cases of early portal thrombosis were found, with long-term follow-up showing that none of the patients developed cavernous transformation of the portal vein. Endoscopy can only observe the degree of esophageal varices, and this study mainly evaluated the risk of rebleeding by observing the formation of portal vein thrombosis. Portal vein thrombosis can only be observed by color Doppler ultrasound of portal vein blood flow. The safety of portal vein thrombosis after splenectomy was evaluated by the above monitoring indicators. ${ }^{19}$

After reviewing the changes in the patient's liver function (Child-Pugh classification), we noticed that the postoperative liver function could basically be maintained at the preoperative level or achieve improvement. The analysis found that the reason might be related to the postoperative increase in hepatic artery blood flow. The following several reasons are considered: (1) the hepatic artery and the portal vein branch become merged into the hepatic sinus, and the postoperative decrease in portal pressure leads to a decrease in hepatic sinus pressure, further causing a corresponding increase in hepatic artery blood flow ${ }^{\mathbf{2 0}}$; (2) the left gastric artery, splenic arteries and common hepatic arteries jointly originate from the celiac trunk. After the splenic arteries and the left gastric artery are ligated, the celiac trunk pressure is relatively constant but the blood flow outflow tract is reduced, resulting in an increase in hepatic artery blood flow; (3) it is reported in the literature that the liver has a regulatory mechanism by the hepatic artery buffering capacity to maintain blood flow stability. When the portal vein pressure drops, the hepatic artery will have compensatory dilation; on the contrary, the hepatic artery blood flow will decrease when the portal vein pressure increases. The liver function is closely associated with the function and status of each hepatocyte. A good nutritional status of the hepatocytes and a rich blood supply can enable the liver function to maintain the preoperative level or even improve it. ${ }^{21}$ In addition, antiviral therapy in patients with hepatitis $\mathrm{B}$ also plays an important role in the postoperative improvement of 
liver function. ${ }^{22}$ This study was mainly based on the clinical manifestations of patients before and after surgery, such as bleeding, liver function, hepatic encephalopathy, etc., to observe the benefits of operation by self-control. ${ }^{23-26}$

Both domestic and foreign reports have shown that the incidence of hepatic encephalopathy after shunting is not high. The rate in the report of Johnson et al. ${ }^{27}$ was $9.6 \%$, and in a domestic report Zhen et al. ${ }^{28}$ cited that the incidence of hepatic encephalopathy was $2.5 \%$ and $4.1 \%$ respectively in 5 and 10 years after devascularization. Our current study of TH gel embolization combined with splenectomy showed that the incidence of hepatic encephalopathy was $2.5 \%$ in 5 years after operation, and $2.5 \%$ in 5-10 years, which was consistent with the relevant research results both at home and abroad, where 9 cases of spleen fever were reported before 2012, with the body temperature decreasing to normal after 4-week symptomatic treatment. After the initiation of operation in 2012, low-molecular weight heparin calcium was conventionally administered as the anticoagulant therapy, involving a total of 8 cases, and no spleen fever occurred again. Of the 73 patients before 2012, cavernous transformation of the portal vein was reported in 16 patients after long-term postoperative follow-up. Therefore, for gastroesophageal varices caused by portal hypertension, gastric coronary venous $\mathrm{TH}$ embolization combined with splenectomy has the advantages of devascularization and maintaining blood perfusion to the liver after surgery. Early postoperative prophylactic administration of low-molecular weight heparin calcium for anticoagulant therapy can effectively prevent splenic fever and portal thrombosis. The low incidence of postoperative hepatic encephalopathy is low, which is of great significance for ensuring postoperative quality of life and longterm survival.

\section{Prospects}

Proper management of portal hypertension in patients with cirrhosis can effectively improve the survival time and quality of life. There is no unified method for the treatment of portal hypertension. Effective control of collateral circulation blood flow and complications is the ultimate benefit of various operations. TH gel blocking the scope of varicose veins, combined with splenic incision to further increase the blood supply of the liver, will bring great benefits to patients. In this study, the operative methods were also further improved. Splenic anatomy in situ and ligation of splenic artery reduced the complications such as bleeding after operation. The blood supply of liver tissue increased further after operation, which greatly reduced the complications of bleeding after operation, and provided better treatment for patients with portal hypertension and varicose veins.

\section{Conclusions}

The procedure of gastric coronary venous embolization with $\mathrm{TH}$ glue in combination with splenectomy for the treatment of cirrhotic portal hypertension and gastroesophageal varices is characterized by a good safety profile, less invasiveness, rapid postoperative recovery, and a lower rebleeding rate, and the subjects achieved a good long-term effect. Therefore, this surgical mode combined with postoperative anticoagulation can achieve better results, and it is an option that can be first considered by patients requiring emergency surgery to stop bleeding or patients with poor liver function, and even some patients of Child-Pugh grade C classification; it also re- mains a relatively ideal surgical method for the current treatment of cirrhotic portal hypertension with gastroesophageal varices.

\section{Acknowledgments}

There is no financial support for this study.

\section{Conflict of interest}

The authors have no conflict of interests related to this publication.

\section{Author contributions}

Study design, patient management, data analysis and writing of the manuscript (ZL, JC, XW), patient recruitment (XW, ZZ, FZ); writing of the manuscript (LS, LL, CC, XW, YJ, QF).

\section{References}

[1] Sauerbruch T, Schierwagen R, Trebicka J. Managing portal hypertension in patients with liver cirrhosis [version 1; peer review: 2 approved]. F1000Research 2018;7(F1000 Faculty Rev):533. doi:10.12688/f1000research.13943.1.

[2] Piekarska A, Berkan EK, Wojcik KM. Surveillance for portal hypertension in the course of liver cirrhosis. Clin Exp Hepatol 2018;4:49-54.

[3] McConnell M, Iwakiri Y. Biology of portal hypertension. Hepatol Int 2018(Suppl 1):11-23. doi:10.1007/s12072-017-9826-x.

[4] Bosch J, Iwakiri Y. The portal hypertension syndrome: etiology, classification, relevance, and animal models. Hepatol Int 2018(Suppl 1):1-10. doi:10.1007/s12072-017-9827-9.

[5] Garbuzenko D V. Contemporary concepts of the medical therapy of portal hypertension under liver cirrhosis. World J Gastroenterol 2015;21(20):6117-6126. doi:10.3748/wjg.v21.i20.6117.

[6] Takamatsu Y, Hori T, Machimoto T, Hata T, Kadokawa Y, Ito T, et al. Intentional modulation of portal venous pressure by splenectomy saves the patient with liver failure and portal hypertension after major hepatectomy: is delayed splenectomy an acceptable therapeutic option for secondary portal hypertension? Am J Case Rep 2018;19:137-144. doi:10.12659/AJCR.907178.

[7] Yang L, Yuan L, Dong R, Yin J K, Wang O, Li T, et al. Two surgical procedures for esophagogastric variceal bleeding in patients with portal hypertension. World J Gastroenterol 2013;19(48):9418-9424. doi:10.3748/wjg.v19.i48.9418.

[8] Hackl C, Schlitt HJ, Renner P, Lang SA. Liver surgery in cirrhosis and portal hypertension. World J Gastroenterol 2016;22(9):2725-2735. doi:10.3748/wjg.v22.i9.2725.

[9] Balducci G, Sterpetti AV, Ventura M. A short history of portal hypertension and of its management. J Gastroenterol Hepatol 2016;31(3):541-545. doi:10.1111/jgh.13200.

[10] Gao DM, He ZS. Direct vision controlled gastric coronary vein embolization for the treatment of portal hypertension. Chin Med 1990;103(12):989-994.

[11] Yin L, Liu H, Zhang Y, Rong W. The surgical treatment for portal hypertension: a systematic review and meta-analysis. ISRN Gastroenterol 2013;2013:464053. doi:10.1155/2013/464053.

[12] Bai DS, Qian JJ, Chen P, Yao J, Wang XD, Jin SJ, et al. Modified laparoscopic and open splenectomy and azygoportal disconnection for portal hypertension. Surg Endosc 2014;28(1):257-264. doi:10.1007/ s00464-013-3182-2.

[13] Zong GQ, Fei Y, Chen J, Liu RM. Selective double disconnection for cirrhotic portal hypertension. J Surg Res 2014;192(2):383-389. doi:10.1016/j.jss.2014.05.065.

[14] Zheng SL. [Clinical effect of cardiac peripheral vascular disconnec- 
tion in emergency treatment of gastroesophageal bleeding caused by portal hypertension]. Zhongguo Xue Xi Chong Bing Fang Zhi Za Zhi 2015;27(2):201-202. doi:10.16250/j.32.1374.2014256.

[15] Al-Bawardy B, Gorospe EC, Saleem A, Buttar NS, Wong KSL. Outcomes and predictors of rebleeding after 2-octyl cyanoacrylate injection in acute gastric variceal hemorrhage. J Clin Gastroenterol 2016;50(6):458-463. doi:10.1097/MCG.0000000000000487.

[16] Rickman OB, Utz JP, Aughenbaugh GL, Gostout CJ. Pulmonary embolization of 2-octyl cyanoacrylate after endoscopic injection therapy for gastric variceal bleeding. Mayo Clin Proc 2004;79(11):1455-1458. doi:10.4065/79.11.1455.

[17] Hermann RE, Henderson JM, Vogt DP, Mayes JT, Geisinger M A, Agnor C. Fifty years of surgery for portal hypertension at the Cleveland Clinic Foundation. Lessons and prospects. Ann Surg 1995;221(5):459-466; discussion 466-468. doi:10.1097/00000658-199505000-00003.

[18] Asoglu O, Ozmen V, Gorgun E, Karanlik H, Kecer M, Igci A, et al. Does the early ligation of the splenic artery reduce hemorrhage during laparoscopic splenectomy? Surg Laparosc Endosc Percutan Tech 2004;14(3):118-121. doi:10.1097/01.sle.0000129397.50124.fa.

[19] Chawla YK, Bodh V. Portal vein thrombosis. J Clin Exp Hepatol 2015;5(1):22-40. doi:10.1016/j.jceh.2014.12.008.

[20] Ho HL, Huang HC. Molecular mechanisms of circulatory dysfunction in cirrhotic portal hypertension. J Chin Med Assoc 2015;78(4):195203. doi:10.1016/j.jcma.2014.10.004.

[21] Li YS, Harn HJ, Hsieh DK, Wen TC, Subeq YM, Sun LY, et al. Cells and materials for liver tissue engineering. Cell Transplant 2013;22(4):685700. doi:10.3727/096368912X655163.
[22] Lao XM, Luo G, Ye LT, Luo C, Shi M, Wang D, et al. Effects of antiviral therapy on hepatitis $B$ virus reactivation and liver function after resection or chemoembolization for hepatocellular carcinoma. Liver Int 2013;33(4):595-604. doi:10.1111/liv.12112.

[23] Kong D, Wang W, Du G, Shi B, Jiang Z, Jin B. Liver retraction using $n$ Butyl-2-Cyanoacrylate (NBCA) glue during laparoscopic splenectomy and azygoportal disconnection in cirrhotic patients. Biomed Res Int 2018;2018:3064046. doi:10.1155/2018/3064046.

[24] Ibrahim M, Mostafa I, Deviere J. New developments in managing variceal bleeding. Gastroenterology 2018;154(7):1964-1969. doi:10.1053/j.gastro.2018.02.023.

[25] Wang Y, Zhan X, Zhu Y, Xie Z, Zhu J, Ye Z. Laparoscopic splenectomy in portal hypertension: a single-surgeon 13-year experience. Surg Endosc 2010;24(5):1164-1169. doi:10.1007/s00464-009-0744-4.

[26] Jiang GQ, Chen P, Qian JJ, Yao J, Wang XD, Jin S J, et al. Perioperative advantages of modified laparoscopic vs open splenectomy and azygoportal disconnection. World J Gastroenterol 2014;20(27):91469153. doi:10.3748/wjg.v20.i27.9146.

[27] Johnson M, Rajendran S, Balachandar TG, Kannan D, Jeswanth S, Ravichandran $\mathrm{P}$, et al. Transabdominal modified devascularization procedure with or without esophageal stapler transection-an operation adequate for effective control of a variceal bleed. Is esophageal stapler transection necessary? World J Surg 2006;30(8):1507-1518; discussion 1519. doi:10.1007/s00268-005-0754-x.

[28] Yang Z, Qiu F. Pericardial devascularization with splenectomy for the treatment of portal hypertension. Chin J Surg 2000;38(9):645-648. doi:10.3760/j:issn:0529-5815.2000.09.001. 